\title{
man \\ A High-Throughput Imagery Protocol to Predict Functionality upon Fractality of Carbon-Capturing Biointerfaces
}

\author{
Bruno Rafael de Almeida Moreira ${ }^{*}{ }^{\dagger}$, Armando Lopes de Brito Filho ${ }^{\dagger}$, Marcelo Rodrigues Barbosa Júnior ${ }^{\dagger}(\mathbb{D}$ \\ and Rouverson Pereira da Silva (iD)
}

check for updates

Citation: Moreira, B.R.d.A.; Brito Filho, A.L.d.; Barbosa Júnior, M.R.; Silva, R.P.d. A High-Throughput Imagery Protocol to Predict Functionality upon Fractality of Carbon-Capturing Biointerfaces. Agronomy 2022, 12, 446. https:// doi.org/10.3390/agronomy12020446 Academic Editor: Silvia Arazuri

Received: 26 December 2021

Accepted: 5 February 2022

Published: 10 February 2022

Publisher's Note: MDPI stays neutral with regard to jurisdictional claims in published maps and institutional affiliations.

Copyright: (C) 2022 by the authors. Licensee MDPI, Basel, Switzerland. This article is an open access article distributed under the terms and conditions of the Creative Commons Attribution (CC BY) license (https:// creativecommons.org/licenses/by/ $4.0 /)$.
Department of Engineering and Mathematical Sciences, School of Agricultural and Veterinarian Sciences, São Paulo State University (Unesp), Jaboticabal, São Paulo 14884-900, Brazil; armando.brito@unesp.br (A.L.d.B.F.); marcelo.junior@unesp.br (M.R.B.J.); rouverson.silva@unesp.br (R.P.d.S.)

* Correspondence: b.moreira@unesp.br

+ These authors contributed equally to this work.

\begin{abstract}
Surface quality is key for any adsorbent to have an effective adsorption. Because analyzing an adsorbent can be costly, we established an imagery protocol to determine adsorption robustly yet simply. To validate our hypothesis of whether stereomicroscopy, superpixel segmentation and fractal theory consist of an exceptional merger for high-throughput predictive analytics, we developed carbon-capturing biointerfaces by pelletizing hydrochars of sugarcane bagasse, pinewood sawdust, peanut pod hull, wheat straw, and peaty compost. The apochromatic stereomicroscopy captured outstanding micrographs of biointerfaces. Hence, it enabled the segmenting algorithm to distinguish between rough and smooth microstructural stresses by chromatic similarity and topological proximity. The box-counting algorithm then adequately determined the fractal dimension of microcracks, merely as a result of processing segments of the image, without any computational unfeasibility. The larger the fractal pattern, the more loss of functional gas-binding sites, namely $\mathrm{N}$ and $\mathrm{S}$, and thus the potential sorption significantly decreases from 10.85 to $7.20 \mathrm{mmol} \mathrm{CO}_{2} \mathrm{~g}^{-1}$ at sigmoid Gompertz function. Our insights into analyzing fractal carbon-capturing biointerfaces provide forward knowledge of particular relevance to progress in the field's prominence in bringing high-throughput methods into implementation to study adsorption towards upgrading carbon capture and storage (CCS) and carbon capture and utilization (CCU).
\end{abstract}

Keywords: adsorbent; box-counting method; high-resolution stereomicroscopy imagery data; physical adsorption; porous carbonaceous material; simple linear iterative clustering algorithm; superpixel segmentation

\section{Introduction}

The IPPC's experts estimate the Earth's surface temperature at $2{ }^{\circ} \mathrm{C}$ above the preindustrial baseline (1850-1900) by 2100. Therefore, stakeholders (e.g., the scientific community, policymakers, and civil community) must be aware of the commitment, cooperation and coordination they need to elaborate on if they are to effectively mitigate global warming and prevent planetary collapse over the coming years [1]. Numerous CCU and CCS platforms exist for reducing emission of $\mathrm{CO}_{2}$. The literature focuses on absorption [2-4], adsorption, cryogenic distillation [5], and separation [6]. Particularly the adsorption works on the simplest physical principles of capturing and binding an adsorbate in pores and functional sites on the surface of the material [7]. Adsorption, relative to absorption on amine-containing solutions, is not energy-intensive and other immediate advantages refer to compactness and conservation of space, and a wider range of suitable scaffoldings and powders to assemble resilient sorbents [8]. The application of carbon-capturing materials is common in coal-firing and gas-firing power stations across the USA, UK and European Union. They can potentially prevent the industry from emitting about 10,000.00 Mt 
$\mathrm{CO}_{2}$ yearly [9]. Precisely, an effective adsorption can remove $85 \%$ of the adsorbate out of the flue-gas consisting of $10-15 \% \mathrm{CO}_{2}$ [9]. However, screening out materials for the purpose of full-scale adsorption can be costly via traditional methodologies, such as adsorption/desorption isotherm [10]. Thus, development of an alternative is imperative to level-up cost-effectiveness of the technology and progress in the field's prominence in bringing CCU and CCS into implementation at a global scale. As adsorbers are often irregular on the surface, an option to analyze them cost-effectively would be an imagery protocol consisting of fractal analysis.

Fractal is the power-law of self-similarity [11]. The theory fundamentally describes anything in nature consisting of similar copies of itself at multiple scales and dimensions, ranging from 2 to 3 [12]. The closer to 2 the $D_{F}$, the smoother the geometric pattern. By contrast, the closer from 3 the $D_{F}$ the rougher and more complex the geometric pattern [13]. Therefore, fractal analytics consists of applying non-traditional mathematics to solve nonEuclidean geometries [14]. It proves useful to analyze adsorption both quantitatively and qualitatively by seamlessly modelling features of a topographically irregular surface $[15,16]$. However, if there is any structural dependency in the dataset, it becomes rather obsolete and thus we may need to approach multifractal analysis [12]. Multifractal analysis handles both accuracy and comprehensiveness. Hence, it can compensate for the non-ability of single fractal analysis to adequately characterize a material for dynamic pore-filling phenomena [12]. For instance, a multifractal model can predict either for distribution of pores upon crushing or adsorption of $\mathrm{CH}_{4}$ on coal with greater precision than possible with the probabilistic functions, namely Gandin-Schumann, Rosin-Rammler and Logit [12]. It provides an overview of the impact of adsorption on the surface's functionality and resilience. If an adsorbate suddenly penetrates through the structure, it destabilizes the adsorber, making it incapable of capturing cost-effectively. By contrast, underperformance is not likely from a gradual adsorption-desorption [12].

The contemporary academic literature about applying fractals to adsorption focuses on fossil resources, such as coal, coalbed methane and shale. By contrast, research and technological development in micro and mesoporous biosurfaces is at an embryonic stage (Figure S1, Supplementary Material). For instance, an original study by Zhang et al. [17] provides a consistent reference of the robustness of fractal numerical simulation in generating data to model a non-Darcy flow in tortuous capillary tubes and decide whether an inorganic porous matrix is feasible to store supercritical $\mathrm{CO}_{2}$. The fractal model, namely Frankel-Halsey-Hill, can predict a functional relationship between adsorption and porosity of a structurally complex and changeable shale [18]. The larger the fractal pore, the more probable the material is to perform an effective adsorption. Furthermore, Zhang et al. [19] on the adsorption of $\mathrm{NO}_{x}$ from flue-gas on sludge char, picture the importance of pore's fractality to the dynamic physical processes of capturing and binding. Most notably, they elaborate how work-up conditions can impact adsorption both negatively and positively. While the pyrolytic residence time does not influence the dimensional features of pore network, such as diameter, width and volume, the temperature can structure-up larger and more complex physical gas-adsorbing sites measurable from an isothermal sorption analyzer.

Numerous analytical protocols exist for determining fractality, for characterization and understanding how it could impact the adsorption. The literature focuses on adsorption/desorption isotherm, AFM [13], photogrammetric reconstruction from SEM [20], mercury intrusion porosimetry, X-ray diffraction, SAXS, SANS, micrographs, and adsorption desulfurization with an auxiliary microwave irradiation [21]. For instance, AFM [13] and SEM [20] can directly measure irregularities on the surface. However, both can limit the field of view to $100 \mu \mathrm{m}$ and, eventually, make the project costly and complex rather than easy-to-setup, besides the requirement of an expert for accuracy and reliability. FESEM, EDS and adsorption/desorption isotherm are an exceptional merger for analyzing pore's morphology and how it impacts the structuration and function of an adsorbing coal [22]. However, the integrative protocol by the authors [22] needs processing micrographs in the 
photogrammetric environment of Image-Pro Plus. The software works on imagery data (2D and 3D). However, addressable and programmable functions such as adjusting size and resolution, cropping and editing specific areas, rotating, adding filters, and balancing often are not available from the open-source version. Another disadvantage refers to the need of polishing samples by an Ar-ion milling system with an accelerating voltage of $6 \mathrm{keV}$ to improve image's quality and resolution, making it time-consuming and energyintensive. Complementarily, another software capable of processing fractal imagery data is MATLAB [23]. The multi-paradigm program supports direct uploads from cameras, scanners, disks or CD-ROM. However, the professional version is private.

An imagery protocol by Ren et al. [24] can determine functional relationships between fractal dimensions of microstructures and relative mechanical stresses on the surface of bituminous coal. It is effective and can process data in real time. However, it can be costly by demanding a universal testing machine, state-of-the-art microcomputer and high-speed camera ( $\sim 50$ FPS) to capture digital images on the material during uniaxial loading at $5 \mathrm{~mm} \mathrm{~min}{ }^{-1}$. Furthermore, the methodology by Lin et al. [25] can predict on BCM for the porosity and how it can impact the adsorption on fractal deep shale with atomic-level precision. The $\mathrm{BCM}$ determines the $D_{F}$ by covering the pattern with boxes then counting the number of boxes touching about it. Graphically, $D_{F}$ is the slope of the curve by plotting the $N_{S}$ on the y-axis against the $s$ on the x-axis. $N_{S}$ and $s$ stand as the number and size of boxes, respectively [26]. Therefore, the steeper the curve, the more fractal the object. By contrast, the flatter the curve, the less fractal the object, as the degree of details does not increase with reducing the box's size. BCM works on a 2D plane by iteratively generating smaller boxes of finer grids until the process achieves the accuracy we might expect on an optimal processing of digital images. Thus, the magnitude of $D_{F}$ ranges from 1 for perfectly smooth geometry to 2 for extremely rough geometry. By shrinking the box's size, we can more accurately capture the pattern. However, if there are any unpredictable low-level properties in the image, $\mathrm{BCM}$ becomes rather complex, making it incapable of determining fractality without computational unfeasibility. An option to improve programmatically determining $D_{F}$ on BCM would be SLIC. SLIC can segment an image into superpixels by chromatic similarity and topological proximity. It can massively generate pixels merely as a result of ' $k$-mean' discretization, so it is likely to prevent BCM from underperformance.

Therefore, in light of research and innovation in applying fractal analysis to adsorption, we elaborated stereomicroscopy, superpixel segmentation and box-counting algorithm into a high-throughput imagery protocol to predict adsorption at microstructural stress. A secondary objective was to analyze if it could be feasible for integrating hydrothermal carbonization and pelletization into a system for making agricultural, agro-industrial and forestry residues into high-throughput carbon-capturing biointerfaces towards progressing on eco-compatible adsorption and waste management.

\section{Materials and Methods}

\subsection{Origination of Scaffoldings in Carbon-Capturing Biointerfaces}

Sources of powders for making carbon-capturing biointerfaces via an integrative carbonization-pelletization system included agricultural, agro-industrial and forestry residues from full-scale sugar-energy mill plant, timber-processing factory, mechanical harvesting and pilot-scale mushroom-growing house (Table 1). Materials were dried at $125{ }^{\circ} \mathrm{C}$ into a constant mass, milled and sieved to $0.25-0.45 \mathrm{~mm}$, then characterized in triplicate to moisture (ASTM E871-82), volatile matter (ASTM E871-82), fixed-carbon (ASTM E871-82), ash (ASTM D1102-84), C, H, O, N, and S (EN 15104, EN 15289) in elemental analyzer (Perkin-Elmer: Waltham, MA, USA, 2400 Series II CHNS/O), and calorific value in an isotherm calorimeter bomb (C 200, IKA-Werke GmbH \& Co. KG: Staufen, Germany), as per the methods in Iftikhar et al. [27]. 
Table 1. Origination and physicochemical properties scaffoldings for making carbon-capturing biointerfaces.

\begin{tabular}{|c|c|c|c|c|c|c|c|c|c|c|c|c|}
\hline Biointerface & Material & Source & Moisture & $\mathbf{V}_{\mathbf{M}}$ & $F_{C}$ & Ash & $\mathrm{C}$ & $\mathbf{H}$ & $\mathrm{O}$ & $\mathbf{N}$ & $S$ & HHV \\
\hline I & $\begin{array}{l}\text { Sugarcane } \\
\text { bagasse }\end{array}$ & $\begin{array}{l}\text { Full-scale sugar-energy } \\
\text { mill plant }\end{array}$ & 12.40 & 77.35 & 14.20 & 8.45 & 46.70 & 10.20 & 42.95 & 0.10 & 0.05 & 19.75 \\
\hline II & $\begin{array}{l}\text { Pinewood } \\
\text { sawdust }\end{array}$ & $\begin{array}{l}\text { Full-scale timber } \\
\text { processing factory }\end{array}$ & 11.40 & 79.80 & 11.70 & 8.50 & 45.20 & 14.85 & 39.80 & 0.10 & 0.05 & 21.40 \\
\hline III & Peanut pod hull & Mechanical harvesting & 71.90 & 62.90 & 6.00 & 31.10 & 50.20 & 2.80 & 46.75 & 0.15 & 0.10 & 15.30 \\
\hline IV & Paddy straw & $\begin{array}{l}\text { Pilot-scale production of } \\
\text { Pleurotus ostreatus }\end{array}$ & 72.20 & 61.50 & 9.35 & 29.15 & 38.40 & 13.40 & 48.10 & 0.05 & 0.05 & 14.45 \\
\hline $\mathrm{V}$ & Peaty compost & $\begin{array}{l}\text { Pilot-scale production of } \\
\text { Agaricus subrufescens }\end{array}$ & 74.55 & 59.05 & 8.65 & 32.30 & 54.10 & 0.60 & 44.90 & 0.20 & 0.20 & 16.10 \\
\hline
\end{tabular}

$\mathrm{V}_{\mathrm{M}}$, volatile matter; $\mathrm{F}_{\mathrm{C}}$, fixed-carbon; $\mathrm{HHV}$; higher heating value.

\subsection{Pilot-Scaling Manufacturing of Carbon-Capturing Biointerfaces}

\subsubsection{Thermochemical Pre-Treatment}

The hydrothermal carbonization (HTC) was carried out in vertical autoclaving reactor (Table S1, Supplementary Material) for ten standard batches. A batch consisted of $15 \mathrm{~kg}$ powdery material as the solid phase for every $150 \mathrm{~L}$ de-ionized water as the catalytical liquid phase, as per the method by Sharma and Dubey [28]. After introducing the load, the equipment was sealed and programmed to $2 \mathrm{~h} \mathrm{HTC}$ at $200{ }^{\circ} \mathrm{C}$; heating ramp was set to $1.5^{\circ} \mathrm{C}$ min $^{-1}$ to a gradual thermochemical pre-treatment, with $\mathrm{N}_{2}$ injected continuously through an automatic gas pump to expel the oxygen inside and consolidate a continuous and porous carbon matrix. The auto-generated pressure inside the reactor was $1.5 \mathrm{MPa}$. After the process leveled off, the equipment was turned off, the pressure valve released and the fraction of hydrochar was recovered, cooled to room temperature in cold water to prevent further coalification. The hydrochar was then filtered through a cellulose-nitrate membrane to remove impurities, heated at $105^{\circ} \mathrm{C}$ into constant mass and stored dry in an airtight receptacle for sampling for pelletization.

\subsubsection{Pressing}

The protocol of compaction started with introducing $1.5 \mathrm{~kg}$ hydrochar into the silo of the assembly of pelleting. The presser machine (Table S2, Supplementary Material) was then set and controlled at $200 \mathrm{MPa}$ and $125{ }^{\circ} \mathrm{C}$ [29] to properly densify the material into market-grade pellets $(\varnothing=7 \mathrm{~mm})$. After grinding, the pelletizer automatically ejected the pellets out of the compressing channel (flat die) directly onto the vibratory screener, and then the conveyor moved them to the storing unit at the end of the process. Samples were cooled to room temperature then stored dry in $2 \mathrm{~kg}$ airtight bags in the laboratory until quantitative analysis.

\subsection{Assessing Potential Adsorption on Biointerfaces}

To quantitatively analyze the impact of material on the ability of the biointerface to capture and bind $\mathrm{CO}_{2}$ in pores and functional sites on the surface, key-variables to adsorption were determined as follows:

The static adsorption of $\mathrm{CO}_{2}\left[\mu, \mathrm{mmol} \mathrm{g}^{-1}\right]$ was determined by thermogravimetric analyzer (PerkinElmer, Waltham, MA, USA); representative samples of $0.1 \mathrm{~g}$ in triplicate were loaded onto the platinum pan and the temperature was raised from 25 to $200{ }^{\circ} \mathrm{C}$, heating ramp and flow rate of $10{ }^{\circ} \mathrm{C} \mathrm{min}{ }^{-1}$ and $50 \mathrm{~mL} \mathrm{~min}^{-1}$, respectively. After the samples were outgassed to drive moisture off, the temperature was decreased to $25^{\circ} \mathrm{C}$ to measure the adsorption on the surface; on the complete saturation, the $\mathrm{CO}_{2}$ was switched to pure $\mathrm{N}_{2}$, and the temperature was increased to $200{ }^{\circ} \mathrm{C}$ to analyze the desorption. The adsorption-desorption cycle was repeated ten times at $1 \mathrm{MPa}$ [30].

Surface area $\left[\mathrm{S}_{\mathrm{A}}, \mathrm{m}^{2} \mathrm{~g}^{-1}\right]$, and diameter $\left[D_{P}, \mathrm{~nm}\right]$ and width $\left[\mathrm{W}_{\mathrm{P}}, \mathrm{nm}\right]$ of pore were determined by Brunauer-Emmett-Teller equation (Equation (1)) and Barrett-Joyner-Halenda method, respectively, from data on adsorption-desorption of $\mathrm{N}_{2}$ on samples of $0.1 \mathrm{~g}$ in 
triplicate in an isothermal sorption analyzer (Q5000 SA, Waters: Milford, MA, USA) at $77 \mathrm{~K}$ [31].

$$
\frac{1}{V_{a}\left(\frac{P_{0}}{P}-1\right)}=\frac{C-1}{V_{m} C} \times \frac{P}{P_{0}}+\frac{1}{V_{m} C}
$$

where $P$ was the partial pressure of adsorbate gas in equilibrium with the surface, $\mathrm{Pa}$; $P_{0}$ was the saturated pressure of adsorbate gas, Pa; $V_{a}$ and $V_{m}$ were the volumes of gas adsorbed at STP and to produce an apparent monolayer on the surface, $\mathrm{mL}$; and $C$ was the dimensionless constant of enthalpic adsorption on the powdery sample.

Volume of pore $\left[V_{P}, \mathrm{~m}^{3} \mathrm{~g}^{-1}\right]$ was also determined from isotherms of $\mathrm{N}_{2}$-sorption on samples of $0.1 \mathrm{~g}$ in triplicate, previously vacuum outgassed at $200{ }^{\circ} \mathrm{C}$ for $6 \mathrm{~h}$ to remove gases or vapors [31].

Durability $[D, \%]$ : The ability for the pellet to resist shocks and collisions was tested in $100 \mathrm{~g}$ samples in triplicate in a custom-made rotating chamber at $50 \mathrm{rpm}$ for $10 \mathrm{~min}$; after the mechanical trial, the material was sieved then weighted to quantify the durable and nondurable (particles finer than $3.25 \mathrm{~mm}$ ) fractions as per the method in Abdulmumini et al. [32].

Hygroscopicity $\left[\mathrm{H}_{\mathrm{yg}}, \%\right]$ : The $\mathrm{H}_{\mathrm{yg}}$ was determined through the ratio of mass of $100 \mathrm{~g}$ samples in triplicate before and after two weeks of storage in a chamber at a relative humidity of $62.25 \pm 1.50 \%$ and temperature of $41.05 \pm 0.70{ }^{\circ} \mathrm{C}$, as per the method in Abdulmumini et al. [32].

Functionality at the surface: To complete analysis and establish an eventual relationship between fractality and functionality, an EDS was carried out on biointerfaces to determine $\mathrm{O}, \mathrm{N}$ and $\mathrm{S}$ at $20 \mathrm{keV}$ for high-resolution scanning [33].

\subsection{Setting-Up Imagery Protocol}

\subsubsection{Stereomicroscopy}

Representative samples of one-hundred random pellets per biointerface were photographed in an apochromatic stereo microscope (ZEISS Stemi-305, Carl Zeiss, Oberkochen, Germany) at $20 \times$ magnification to maximize the field of view and acquire $18 \mathrm{~mm}$ objects. The scanning was performed in a routine work lab in a dark room to prevent light from damaging images and thus ensure outstanding contrast and color accuracy. Cylindric pellets were placed horizontally on the plate then $90^{\circ}$ flipped to capture the whole surface through interchangeable confocal objective lenses and thus gather sufficient details on lowlevel regions for photogrammetric reconstruction of fractality from complete micrographs without cropping, rotating, balancing, filtering or any other clever feature for adjusting files. Pixel intensity was programmatically extracted from snapshots of 8 bits in the environment of ZEN software then imported as an Excel file or notepad extension to computationally determine the $D_{F}$ of microcracks. Snapshots were saved as PNG, which is a raster-graphic format file supporting lossless data compression and compatible with soft computing.

\subsubsection{Superpixel Segmentation and Box-Counting}

To computationally determine the $D_{F}$ (Equation (2)) of mechanical stresses on biointerfaces upon micrographs, we first performed an oversegmentation with SLIC algorithm to group images into superpixels by color similarity and topological proximity merely as a result of processing low-intensity regions in the CIELAB space. The BCM was then applied to sub-graphs which carry more information than pixels and align better with edges than rectangular patches. To photogrammetrically reconstruct the biointerfaces' surface into line charts, fractal superpixels were quantized and organized in histograms by intensity, and then the frequency of observations was normalized between 1 and 2 to refer the thresholds of BCM for smoothness and roughness, respectively.

$$
D_{F}=\lim _{\delta \rightarrow \infty} \frac{\ln \left(N_{s}\right)}{\ln (s)}
$$


where $N_{S}$ was the number of boxes necessary to completely cover the pattern, and $s$ was the box size [34].

\subsection{Data Analysis}

A box-plot diagram was elaborated to compare biointerfaces by fractality. To predict functionality upon fractality, sigmoid Gompertz function (Equation (3)) was fitted to data, starting parameterization with $\alpha=10, \beta=1$ and $k=0.10$ [35]; its adequacy was analyzed on adjusted coefficient of determination $\left(\mathrm{r}_{\mathrm{adj}}{ }^{2}\right)$. Spearman correlation was measured between independent variables. Another method of applying explanatory statistics included PCA to orthogonalize the high-dimensionality original dataset into useful latent hits with eigenvalues equal to 1 or greater by KMO test [36]. All analyses were carried out in the environment of R-project for statistical computing and graphics [37].

$$
f_{x}=\alpha e^{-\beta e^{-k x}}
$$

where $f_{x}$ was the dependent variable; $x$ was the independent variable; $\alpha$ was the asymptote; $\beta$ was the infection point; $\kappa$ was the slope coefficient, and $e$ was the Euler constant.

\section{Results}

\subsection{Fractality of Biointerfaces}

Apochromatic stereomicroscopy enabled us to capture outstanding snapshots on biosurfaces (Figure 1). We required 45-60 min to process a single sample of one-hundred pellets. The SLIC algorithm segmented the micrographs into k-connected superpixels merely as a result of processing low-intensity regions in the image by color similarity and topological proximity. Its computational power and robustness towards discretization generated sufficient information for pixel-wise quantization of derivative site-specific RGB microarrays by intensity of color or hue. Hence, superpixel oversegmentation enabled the BCM to accurately capture the $D_{F}$ of microstructural stresses on biosurfaces and photogrammetrically reconstruct them by the fractality (Figure 2).
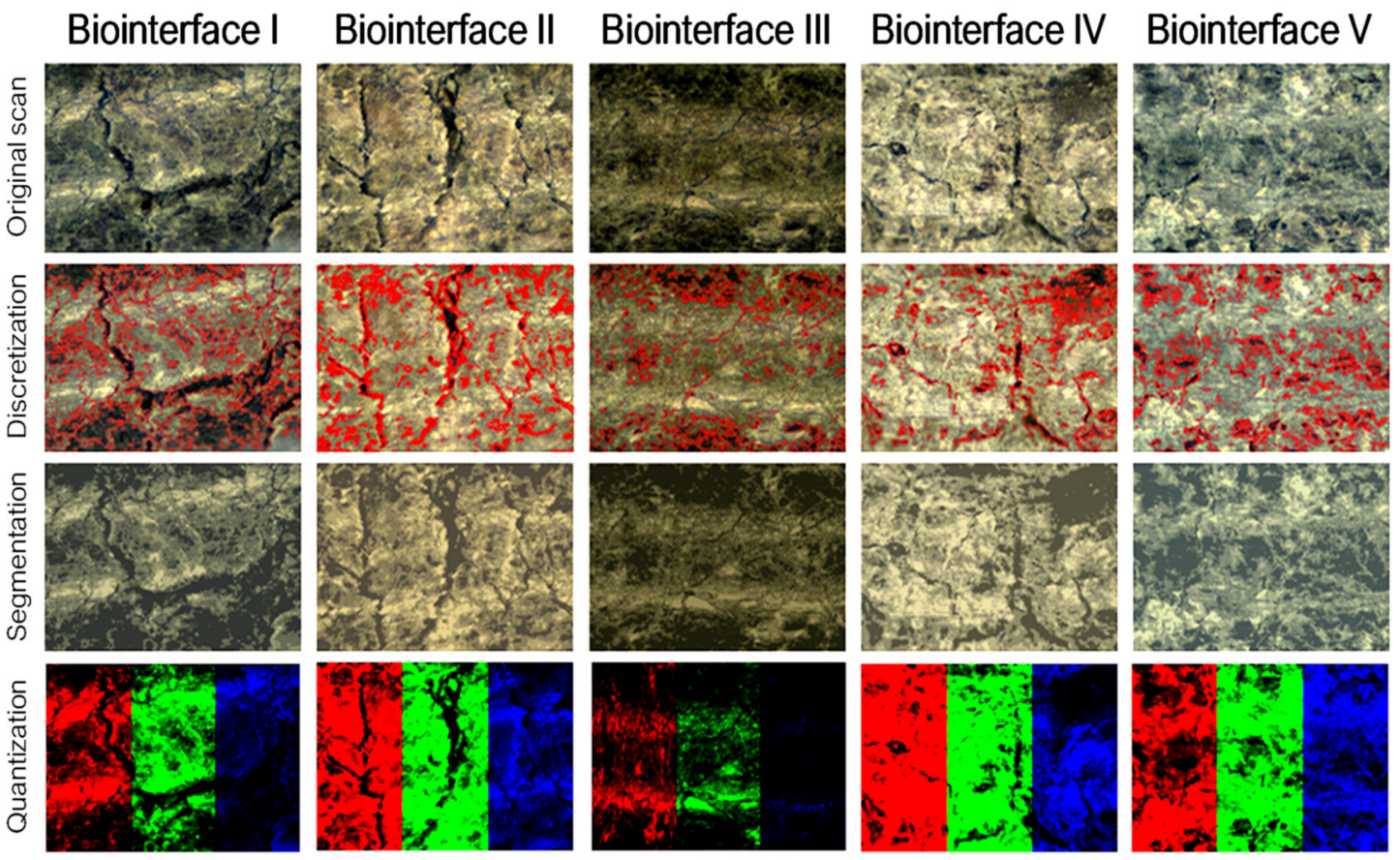

Figure 1. Computational processing of micrographs on fractal carbon-capturing biointerfaces from hydrochars of sugarcane bagasse (I), pinewood sawdust (II), peanut pod hull (III), wheat straw (IV) and peaty compost $(\mathrm{V})$. The outcome of apochromatic stereomicroscopy, namely original scan, becomes 
the input for segmentation. The SLIC algorithm then discretizes between rough and smooth regions on the digital surface merely as a result of processing low-intensity properties in the 2D plane. The next step of the protocol is to measure the intensity of k-mean pixel-wise segments of an image (RGB quantization, range $0-255$ ) for determination of $D_{F}$ on box-counting algorithm and photogrammetric reconstruction of fractality into a descriptive diagram (box-plot).

Biointerface I

$0 \quad 12525037550062575087510001125125013751500162517501875$

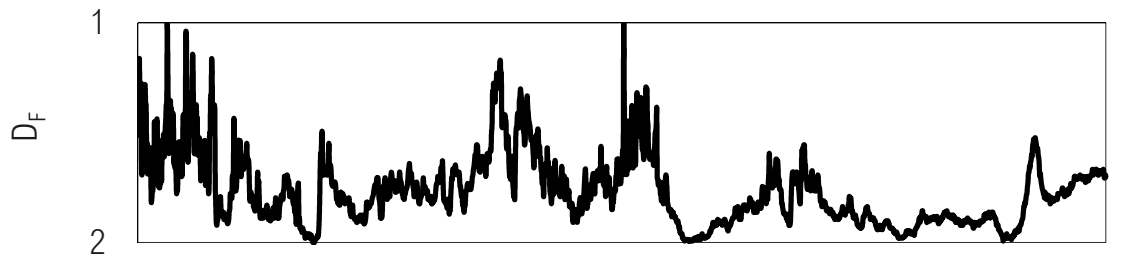

Biointerface II

$0 \quad 12525037550062575087510001125125013751500162517501875$

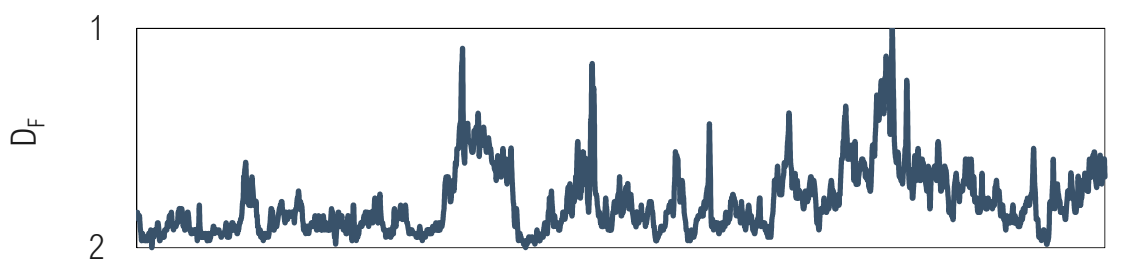

Biointerface III

$0 \quad 12525037550062575087510001125125013751500162517501875$

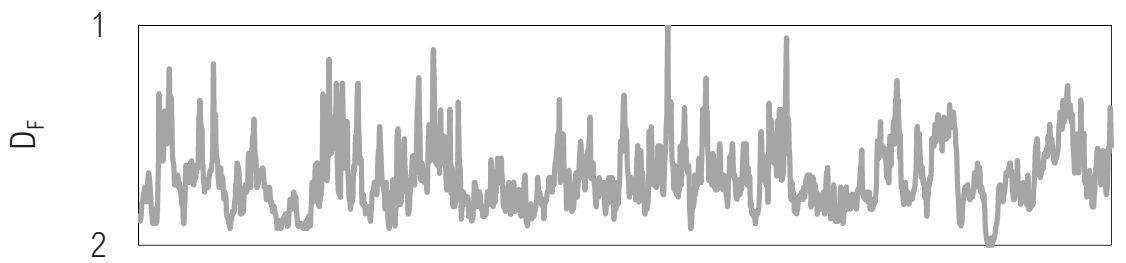

Biointerface IV

$0 \quad 12525037550062575087510001125125013751500162517501875$

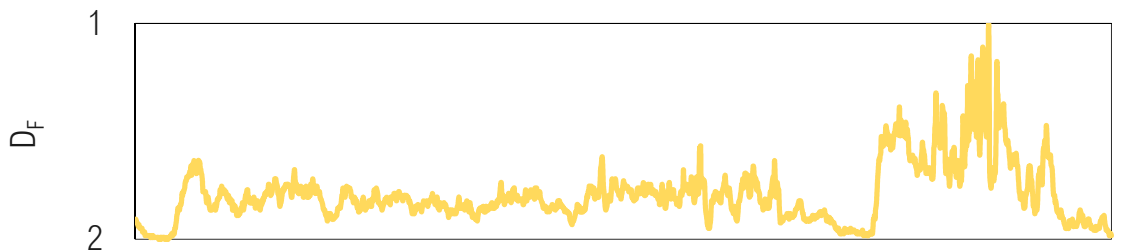

Figure 2. Cont. 
$0 \quad 12525037550062575087510001125125013751500162517501875$

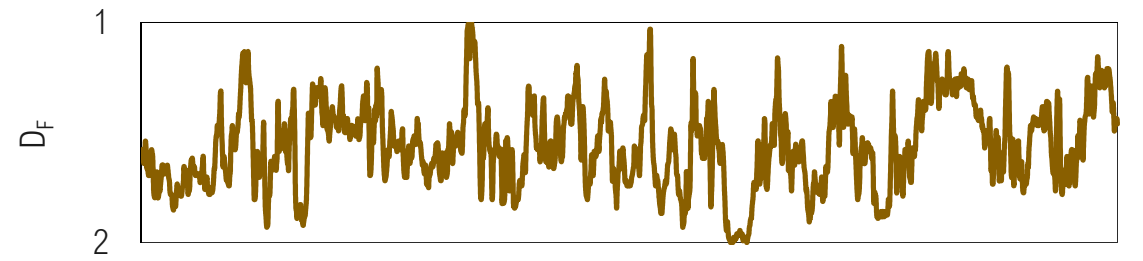

Figure 2. Photogrammetric distribution of fractal microcracks on carbon-capturing biointerfaces from hydrochars of sugarcane bagasse (I), pinewood sawdust (II), peanut pod hull (III), wheat straw (IV) and peaty compost $(\mathrm{V})$. The closer to 1 the $D_{F}$, the smoother the pattern. By contrast, the closer from 2 the $D_{F}$ the rougher the pattern.

Plainly, fractality depended on typology of feedstock for making hydrochar into an adsorbent (Figure 2). Thereby, inter-quartile range $\left(\mathrm{Q}_{75}-\mathrm{Q}_{25}\right)$ of $D_{F}$ was $1.40-1.70$ for the biointerface $\mathrm{V}$ (peaty compost), making it the smoothest and most homogeneous high-throughput carbon-capturing framework. Another smoother bioproduct referred to biointerface III (peanut pod shell) by developing microstructural stresses with $D_{F}$ of 1.60-1.795. By contrast, lower $\left(\mathrm{Q}_{25}\right)$ and upper $\left(\mathrm{Q}_{75}\right)$ quartiles of $D_{F}$ were, respectively, 1.75 and 1.85 for the biointerface IV (wheat straw), making it the roughest and most heterogeneous biostructure by concentrating more points outside the range of "whiskers" in the box-plot (Figure 3). Additionally, sugarcane bagasse and pinewood sawdust developed topographically similar biointerfaces, with microcracks in the inter-quartile ranges of $D_{F}$ of $1.695-1.895$ and $1.70-1.90$, respectively.

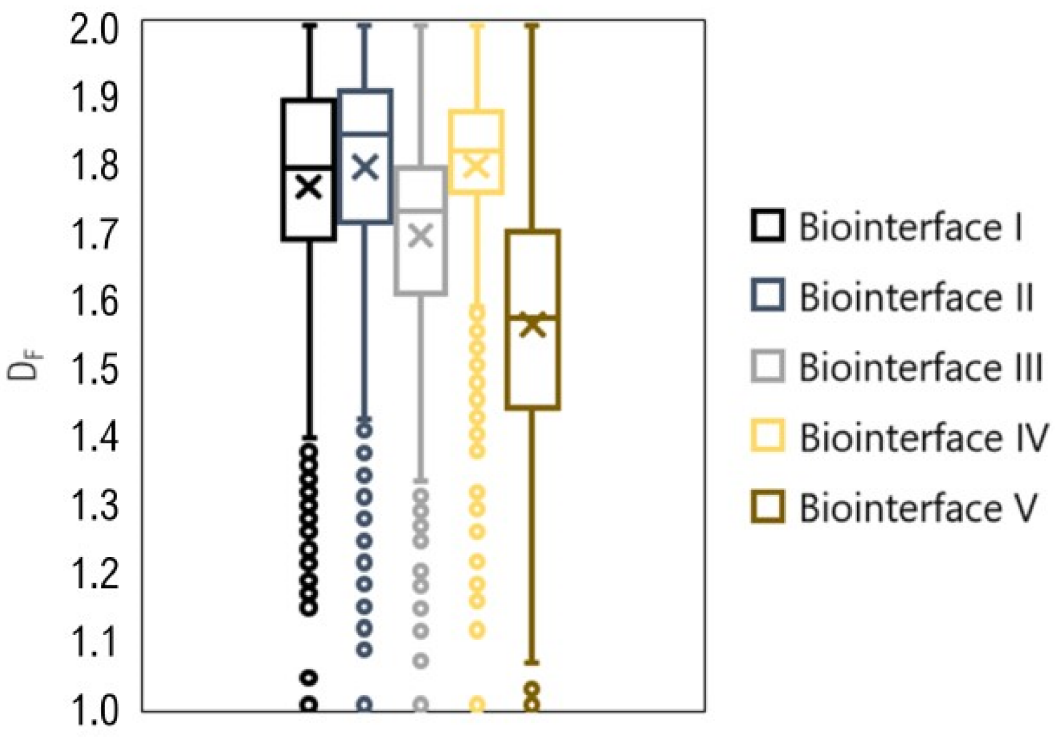

Figure 3. Heterogeneity of fractal mechanical stresses on carbon-capturing biointerfaces from hydrochars of sugarcane bagasse (I), pinewood sawdust (II), peanut pod hull (III), wheat straw (IV) and peaty compost $(\mathrm{V})$. The larger the box, the more heterogeneous the surface. By contrast, the shorter the box, the more homogeneous the surface. Any point outside the range of "whiskers" is an indicator of non-random variability.

Overall, the imagery protocol we propose to analyze topographically irregular carbonadsorbing frameworks proved useful to determine the fractality and how it could impact the functionality at the surface. Although we successfully harmonized stereomicroscopy, SLIC and BCM into a high-throughput protocol, it consumed an appreciable amount of 
time to process samples and thus automation is necessary for overcoming the operational limitation to scale.

\subsection{Potential Adsorption on Biointerfaces}

All fractal biointerfaces proved useful for high-throughput adsorption (Table 2). However, biointerface $\mathrm{V}$ (peaty compost) most effectively adsorbed the $\mathrm{CO}_{2}$. Hence, it outstripped the other potential physical sorbents in the process of capturing and binding molecules of adsorbate to pores and at functional sites on the surface. Biointerface $\mathrm{V}$ structured up the largest inherent pores and contained most gas-binding sites, namely $\mathrm{N}$ and S. In addition, it ended up "less fractally" in microstructural stresses and thus most resisted loss of functionality by mechanical deformation and absorption of environmental vapor during storage. Biointerfaces I (sugarcane bagasse) and III (peanut pod shell) encapsulated the $\mathrm{CO}_{2}$ as effectively as the distinctive biointerface $\mathrm{V}$, although they developed larger non-original irregularities and contained more $\mathrm{O}$, which likely were characteristics of disordering at the surface. By contrast, biointerfaces II (pinewood sawdust) and IV (wheat straw) developed the largest fractal microcracks and therefore were the roughest on the finish surface. Mechanical stresses heavily penetrated through them and promoted critical breakpoints, hygroscopicity and, most importantly, losses of functional gas-binding alkaline sites ( $\mathrm{N}$ and $\mathrm{S}$ ) and matter $(\mathrm{C})$. Since roughness destabilized the material, we could track significant negative correlations between $D_{F}$ and $\mu(\mathrm{r}=-0.75), \mathrm{C}(\mathrm{r}=-0.85), \mathrm{N}$ $(\mathrm{r}=-0.90), \mathrm{S}(\mathrm{r}=-0.95)$, however, a significant positive correlation with $\mathrm{H}_{\mathrm{yg}}(\mathrm{r}=0.95)$ (Table S3, Supplementary Material).

Table 2. Static adsorption and physicochemical properties of biointerfaces.

\begin{tabular}{|c|c|c|c|c|c|}
\hline \multirow{2}{*}{ Property } & \multicolumn{5}{|c|}{ Biointerface } \\
\hline & I & II & III & IV & $\mathbf{V}$ \\
\hline Static adsorption $\left[\mathrm{mmol} \mathrm{CO} \mathrm{Cg}^{-1}\right]$ & 8.20 & 7.45 & 8.35 & 7.10 & 8.65 \\
\hline Surfac area $\left[\mathrm{m}^{2} \mathrm{~g}^{-1}\right]$ & 98.10 & 97.95 & 103.80 & 88.35 & 105.40 \\
\hline Width of pore $[\mathrm{nm}]$ & 1.20 & 1.05 & 1.60 & 1.10 & 1.65 \\
\hline Diameter of pore [nm] & 1.80 & 1.65 & 2.20 & 1.35 & 2.25 \\
\hline Volume of pore $\left[\mathrm{m}^{3} \mathrm{~g}^{-1}\right]$ & 70.05 & 60.80 & 95.10 & 55.40 & 105.60 \\
\hline$C[\%]$ & 46.70 & 45.20 & 50.20 & 38.40 & 54.10 \\
\hline $\mathrm{O}[\%]$ & 42.95 & 39.80 & 46.75 & 48.10 & 44.90 \\
\hline $\mathrm{N}[\%]$ & 0.10 & 0.10 & 0.15 & 0.05 & 0.20 \\
\hline $\mathrm{S}[\%]$ & 0.05 & 0.05 & 0.10 & 0.05 & 0.20 \\
\hline Hygroscopicity [\%] & 8.10 & 8.35 & 7.80 & 9.50 & 5.45 \\
\hline Durability [\%] & 97.90 & 96.60 & 98.05 & 97.05 & 99.10 \\
\hline Fractal dimension of microstructural stress & 1.75 & 1.75 & 1.70 & 1.80 & 1.55 \\
\hline
\end{tabular}

Sugarcane bagasse (I), pinewood sawdust (II), peanut pod hull (III), wheat straw (IV) and peaty compost (V).

\subsection{Adsorption-Fractality Nexus}

The sigmoid Gompertz function adequately predicted the adsorption upon fractality of microstructural stresses on carbon-capturing biointerfaces (Figure 4). Since biointerface $\mathrm{V}$ (peaty compost) developed the smoothest and most homogeneous surface, it is associated with the most gradual breakthrough curves in physicochemical losses of $\mathrm{C}, \mathrm{N}$ and $\mathrm{S}$, and adsorption. Thereby, we could estimate (Table S4, Supplementary Material) the lowest value for the shape-parameter $k$ to $\mu$ of biointerface $V$, further supporting its distinct behavior. Most notably, revealing how the magnitude of fractal stress could negatively impact the adsorption by decreasing availability of functional alkaline sites for the adsorbate to have contact with the surface. 

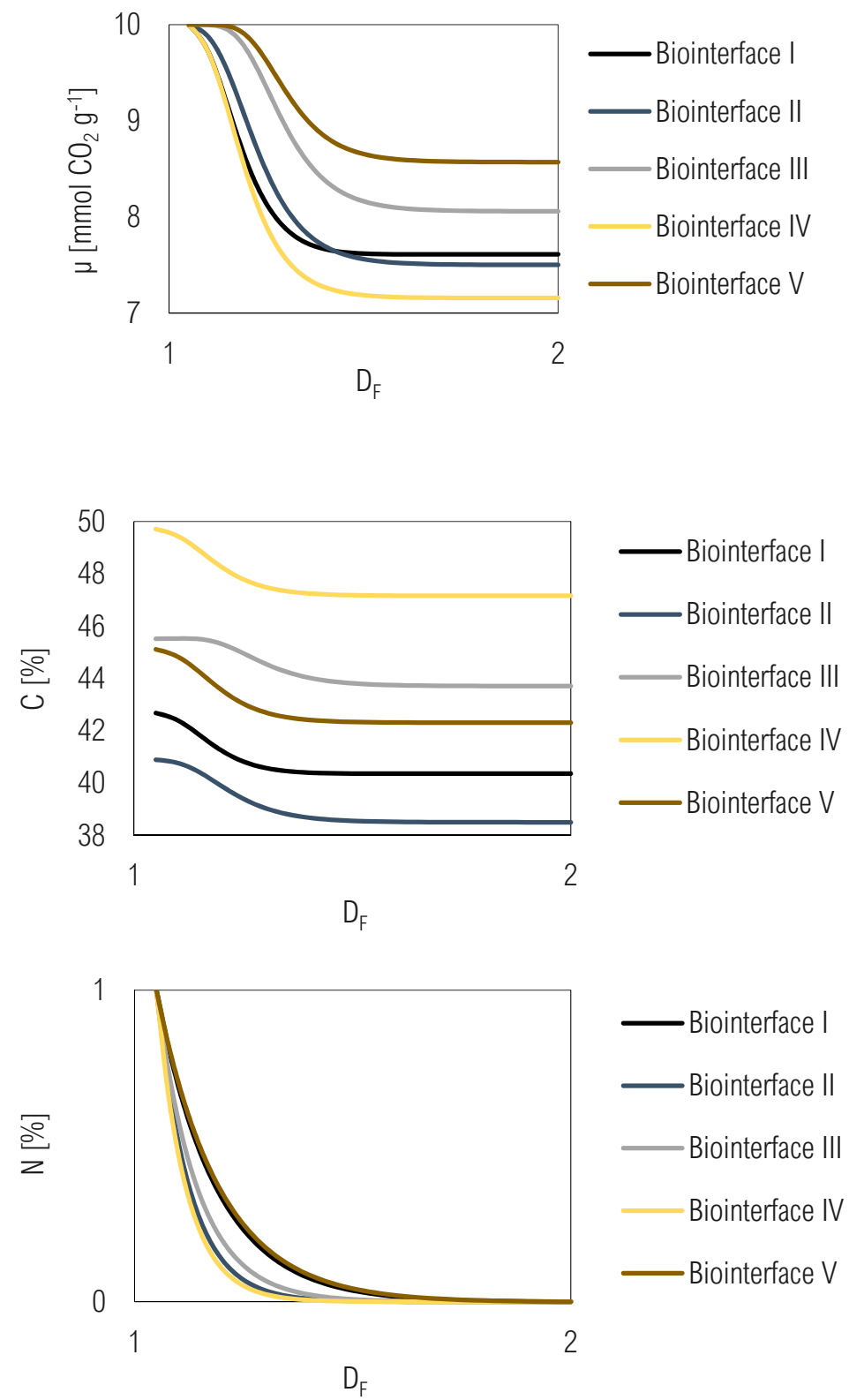

—Biointerface I

_ Biointerface II

_ Biointerface III

Biointerface IV

—Biointerface V

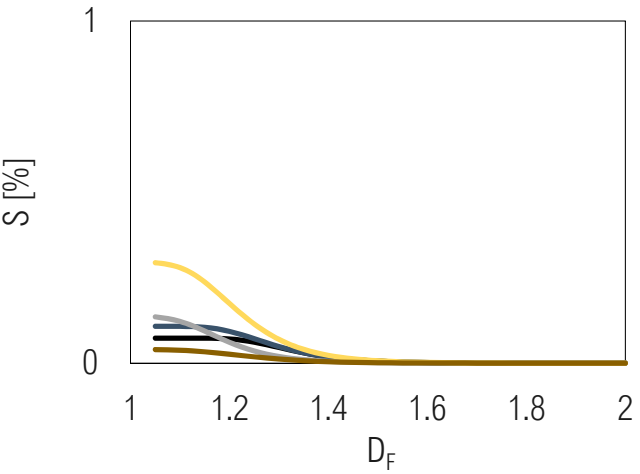

—Biointerface I

— Biointerface II

_Biointerface III

- Biointerface IV

_ Biointerface V

Figure 4. Functional relationships between adsorption, functionality and fractality of bioterfaces from hydrochars of sugarcane bagasse (I), pinewood sawdust (II), peanut pod hull (III), wheat straw (IV) and peaty compost (V) at sigmoid Gompertz function.

The flatter the curve, the less probable the material is to a functionality-limiting fractality. By contrast, the steeper the curve, the more probable the fractal stress is to 
disorder the function of an adsorbent. We could capture the limitation of rougher fractals to functionality into estimates of $\beta>1$ for N. A $\beta \geq 1$ accelerates the relative decrease in functionality with irregularity. By contrast, $\beta<1$ decelerates the relative decrease, while the expectation for $\beta=1$ is to keep it constant. Largest values for both $\beta\left(2.75 \times 10^{-4}\right)$ and $k(-6.35)$ for the $S$ on biointerface IV (wheat straw) validated the importance of avoiding fractals as large as 1.70-1.90 for an adsorbent to properly function.

Overall, we could decide 1.40 as the limit of $D_{F}$ of power-law microstructural stresses to develop high-throughput carbon-capturing frameworks, capable of $8.65 \mathrm{mmol} \mathrm{g}{ }^{-1}$. Any value above 1.40 could not change the functionality. However, Gompertz model always has the limitation of not fitting for the stationary region of a sigmoidal dynamics.

\subsection{Cross-Validation of Protocol}

The PCA robustly divided the high-dimensionality dataset into useful subsets (Table S5, Supplementary Material). The orthogonal latent hits, namely $\mathrm{PC}_{\mathrm{I}}$ and $\mathrm{PC}_{\mathrm{II}}$, together explained about $90 \%$ of variability in functionality-fractality nexus of biointerfaces capable of adsorption and chemisorption of $\mathrm{CO}_{2}$. The $\mathrm{PC}_{\mathrm{I}}$ (bifunctionality) had significant positive correlations with $\mu(\mathrm{r}=0.90), \mathrm{S}_{\mathrm{A}}(\mathrm{r}=0.90), \mathrm{W}_{\mathrm{P}}(\mathrm{r}=0.95), D_{P}(\mathrm{r}=0.90), V_{P}(\mathrm{r}=0.95), \mathrm{C}$ $(r=0.95), N(r=0.95), S(r=0.90)$ and durability $(r=0.90)$, however, significant negative correlations with $(\mathrm{r}=-0.95)$ and $D_{F}(\mathrm{r}=-0.95)$. The $D_{F}$ and $\mathrm{H}_{\mathrm{yg}}$ moved towards the left-lower and left-upper quadrants in the bi-plot chart, respectively, closest to structuration of lowest-intensity pixels (Figure 5; Figure S2, Supplementary Material). Therefore, the larger the non-Euclidean failure on the surface the lower the intensity of pixel, and thus the more likely the biointerface is to not properly function. Biointerface V (peaty compost) most concentrated high-intensity pixels in 2D plane, according to its distinct capability to adsorb $\mathrm{CO}_{2}$. Complementarily, $\mathrm{PC}_{\mathrm{II}}$ (disordering at the surface) had a single significant positive correlation with $\mathrm{O}(\mathrm{r}=0.95)$. The $\mathrm{O}$ moved towards the right-upper quadrant in the factorial map, closest to low-intensity pixels outside the interquartile range of $D_{F}$. Therefore, the more topographically irregular and heterogeneous the surface the higher the content of $\mathrm{O}$, as the larger the loss of hetero atoms of $\mathrm{N}$ and S. Biointerface IV (wheat straw) produced more low-intensity and medium-intensity pixels than any other biomaterial, which were characteristics of rougher and more oxygenated adsorbent with lower durability and not capable of performing an effective adsorption. Plainly, an atypical oxygenation in biointerface IV disordered its potential adsorption.

\section{Discussion}

We created an imagery protocol to predict adsorption in the fractal dimension of microstructural stresses. Most notably, we validated it by analyzing carbon-capturing biointerfaces. The protocol of high-resolution stereomicroscopy can be useful to capture outstanding chromatic contrast at biosurfaces and generate sufficient imagery data in micrographs to determine the $D_{F}$ on BCM after pixel-wise segmentation and quantization. The SLIC algorithm can perform an excellent oversegmentation by color or hue similarity and topological proximity in 2D plane, and thus enable the BCM to adequately determine the $D_{F}$ of irregular microtropographies on digital biosurfaces. The outputs of BCM are accurate inputs to describe on sigmoid Gompertz function the dynamics between fractality and adsorbent's overall quality. Our approach is self-sustaining and could compensate for the complexities of traditionally determining the $D_{F}$ in sorption analyzer or thermogravimetric equipment. The user can quantitatively analyze adsorbents easily and, potentially, save some amount of time pre-processing samples to improve imagery data quality. Another advantage, relative to the existing literature [12,23], is the independence from private analytical softwares. The R-project is an open-sourcing programming language and supports cutting edge computer vision. Disadvantages of our concept are few. However, snapshotting could be time-consuming, which emphasizes the necessity of automation before resembling conditions on an industrial scale. Another direction for future studies of the soundness of our preliminary yet exciting approach is to analyze it economically. 

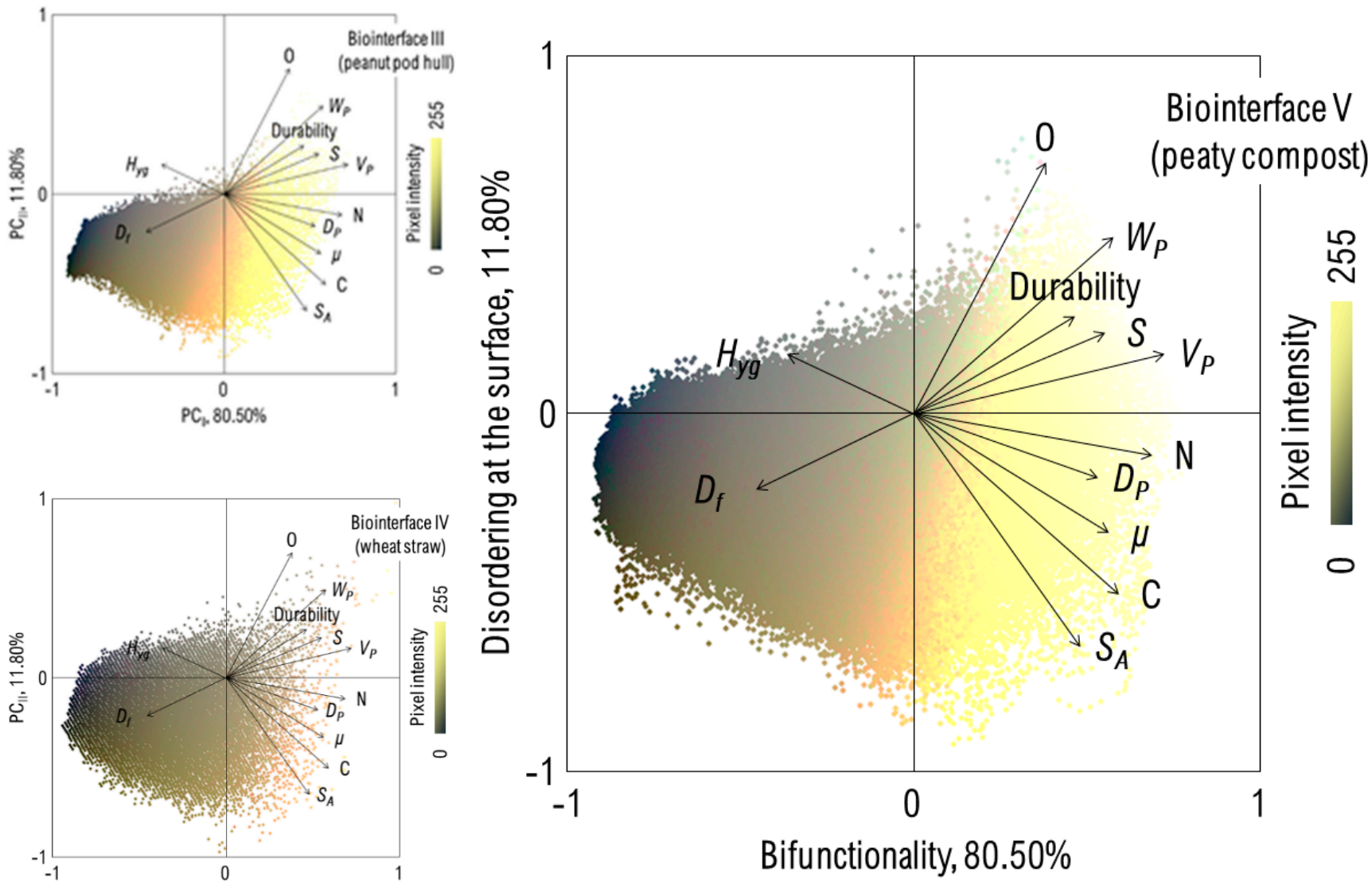

Figure 5. Functionality-fractality nexus. The factorial diagram predicts the functionality upon imagery data. For instance, the lower the intensity of pixel the rougher the mechanical stress, and thus the more intensive the loss of structural pores and functional sites to an effective capturing and binding of $\mathrm{CO}_{2}$ on the surface of the material.

Biointerface V can distinctly develop "less fractally" mechanical stresses, preserving most of its original physics and chemistry of surface to support an effective capturing and binding of acidic $\mathrm{CO}_{2}$ in pores and at alkaline functional sites. If $D_{F}$ is larger than 1.40 , which corresponds to power-law stresses on the highest carbon-capturing biointerface, fractal microcracks can promote significant physical and chemical losses, considerably declining adsorption. If not, underperformance is not likely. Similar studies on gas-adsorbing biocarbons are often not available from the typical literature, making it challenging for contrasting our trends. However, we can read into a few examples where the larger either pore or edge of fractal dimension, the higher the adsorption $[19,25]$, supporting the outperformance of biointerface V. Peaty compost can develop larger and more regular pores, relative to other raw materials. Hence, it is the most reliable option to convert low-pressure powders into high-performance carbon-capturing frameworks. Another potential option to develop high-performance physical biosorbents would be peanut pod hull. Recycling or re-use of both peaty compost and peanut pod hull could create conditions for promoting carbon-to-waste paths across plans to green chemistry, circular economy and net zero society. Biointerfaces could replace fossil fuels in adsorption, potentially improving its environmental footprint. Thus, further in-depth investigations must focus on LCA for assessing if it would be possible for our approach to reduce environmental impacts of developing adsorbers towards CCS and CCU platforms.

Sigmoid Gompertz function and PCA can be of great assistance to accurately predict how the magnitude and distribution of fractals on the surface can impact the characteristics of both imagery protocol and product. Sridhar et al. [38] harmonized the features of 
adsorption and fractality of pores. Yet, our study can bring innovation in extracting useful statistics from non-Euclidean geometries non-inherent to the biomaterial to describe the destabilization of pore network architecture, hetero atoms of $\mathrm{N}$ and $\mathrm{S}$, and hydrophobicity. Ren et al. [24] stressed the capacity of an atypical hygroscopicity to destroy functional sites on the surface, thereby reducing adsorption, in agreement with the negative correlations between $D_{F}, \mu$ and $\mathrm{H}_{\mathrm{yg}}$. Larger fractals can make it easier for the surface to uptake water, reducing adsorption by promoting either aging on pores and physicochemical functional sites or further structural damage. An indicative of disordering at the surface is an accentuated concentration of $\mathrm{O}$, which is predictable by orthogonalizing lower-intensity pixels in micrographs. An atypical oxygenation is attributable to rougher microcracks at the level of adsorbent or, correspondently, lower-intensity pixels at the level of scanning.

\section{Conclusions}

Our study introduces a high-throughput imagery protocol to analyze functionality upon fractality of carbon-capturing biointerfaces. By integrating image processing and fractal analytics, we are able to obtain typical micrographs to determine the fractality of microstructural stress and how it impacts the functionality of an adsorbent. The larger the fractal dimension of an atypical irregularity on the surface, the lower the potential physical sorption, as the loss of functional gas-binding sites and matter occurs. Our insights are timely and serve as a springboard of developing cutting edge analytical methods of adsorption. In addition, carbon-capturing biointerfaces act as an opening for ecocompatible solutions to progress in the field's prominence in evolving CCS and CCU platforms towards mitigating global warming without negatively impacting on the society and environment.

Supplementary Materials: The following supporting information can be downloaded at: https: / / www.mdpi.com/article/10.3390/agronomy12020446/s1, Figure S1: Bibliometric network on the specific topic of 'applying fractals to adsorption' (Elsevier's Scopus and Clarivate's Web of Sciences on 1 December 2021); Figure S2: Functional relationship between properties of fractal carbon-capturing biointerfaces and pixel-wise stereomicroscopy imagery data; Table S1: Specifications of digital autoclave; Table S2: Specifications of automatic pelletizer; Table S3: Spearman matrix for relationships between properties of fractal carbon-capturing biointerfaces; Table S4: Parametrization and adequacy of sigmoid Gompertz function for adsorption of $\mathrm{CO}_{2}$ on fractal biointerfaces; Table S5: Principal components into the adsorption-fractality nexus.

Author Contributions: Conceptualization, B.R.d.A.M.; methodology, B.R.d.A.M.; formal analysis, B.R.d.A.M.; investigation, B.R.d.A.M., A.L.d.B.F. and M.R.B.J.; data curation, B.R.d.A.M.; writingoriginal draft preparation, B.R.d.A.M.; writing-review and editing, B.R.d.A.M., A.L.d.B.F. and M.R.B.J.; visualization, B.R.d.A.M., A.L.d.B.F., M.R.B.J. and R.P.d.S.; supervision, R.P.d.S.; project administration, R.P.d.S.; funding acquisition, R.P.d.S. All authors have read and agreed to the published version of the manuscript.

Funding: This research was funded by Coordination for the Improvement of Higher Education Personnel (CAPES, financing code 001) and Coordination of the Graduate Program in Agronomy, Plant Production (FCAV/Unesp).

Institutional Review Board Statement: Not applicable.

Informed Consent Statement: Not applicable.

Data Availability Statement: The data that support the findings of this study are available on request from the corresponding author, B.R.d.A.M.

Conflicts of Interest: The authors declare no potential conflict of interest. 


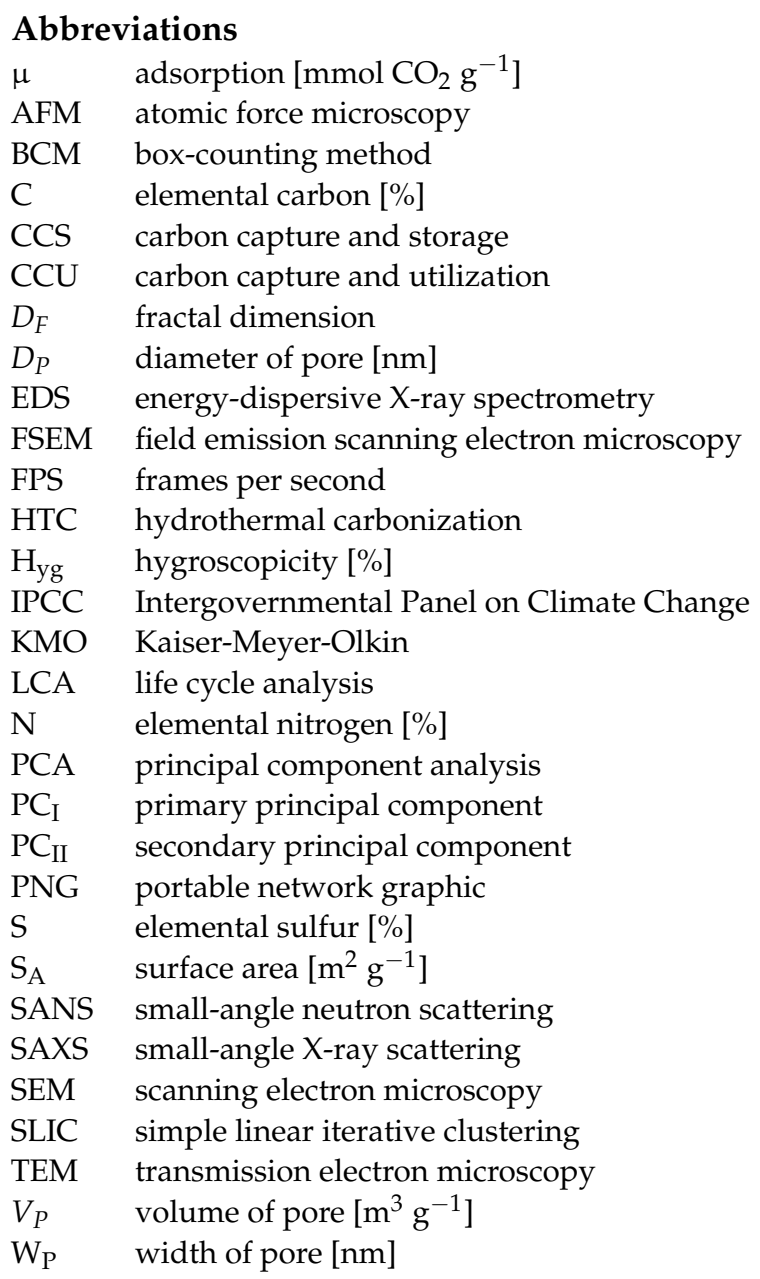

\section{References}

1. Masson-Delmotte, V.P.; Zhai, A.; Pirani, S.L.; Connors, C.; Péan, S.; Berger, N.; Caud, Y.; Chen, L.; Goldfarb, M.I.; Gomis, M.; et al. (Eds.) Sixth Assessment Report Climate Change 2021: The Physical Science Basis-A Report of Working Group I to the Sixth Assessment Report of the Intergovernmental Panel on Climate Change; IPCC: Geneva, Switzerland, 2021.

2. Warudkar, S.S.; Cox, K.R.; Wong, M.S.; Hirasaki, G.J. Influence of stripper operating parameters on the performance of amine absorption systems for post-combustion carbon capture: Part, I. High pressure strippers. Int. J. Greenh. Gas. Control. 2013, 16, 342-350. [CrossRef]

3. Trickett, C.A.; Helal, A.; Al-Maythalony, B.A.; Yamani, Z.H.; Cordova, K.E.; Yaghi, O.M. The chemistry of metal-organic frameworks for $\mathrm{CO}_{2}$ capture, regeneration and conversion. Nat. Rev. Mater. 2017, 2, 17045. [CrossRef]

4. Minelli, M.; Papa, E.; Medri, V.; Miccio, F.; Benito, P.; Doghieri, F.; Landi, E. Characterization of novel geopolymer-Zeolite composites as solid adsorbents for $\mathrm{CO}_{2}$ capture. Chem. Eng. J. 2018, 341, 505-515. [CrossRef]

5. Coenen, K.; Gallucci, F.; Hensen, E.; van Sint Annaland, M. Kinetic model for adsorption and desorption of $\mathrm{H}_{2} \mathrm{O}$ and $\mathrm{CO}_{2}$ on hydrotalcite-based adsorbents. Chem. Eng. J. 2019, 355, 520-531. [CrossRef]

6. Kacem, M.; Pellerano, M.; Delebarre, A. Pressure swing adsorption for $\mathrm{CO}_{2} / \mathrm{N}_{2}$ and $\mathrm{CO}_{2} / \mathrm{CH}_{4}$ separation: Comparison between activated carbons and zeolites performances. Fuel Process. Technol. 2015, 138, 271-283. [CrossRef]

7. Lillia, S.; Bonalumi, D.; Fosbøl, P.L.; Thomsen, K.; Jayaweera, I.; Valenti, G. Thermodynamic and kinetic properties of $\mathrm{NH}_{3}$ $\mathrm{K}_{2} \mathrm{CO}_{3}-\mathrm{CO}_{2}-\mathrm{H}_{2} \mathrm{O}$ system for carbon capture applications. Int. J. Greenh. Gas Control 2019, 85, 121-131. [CrossRef]

8. Igalavithana, A.D.; Kim, K.-H.; Jung, J.-M.; Heo, H.-S.; Kwon, E.E.; Tack, F.M.G.; Tsang, D.; Jeon, Y.J.; Ok, Y.S. Effect of biochars pyrolyzed in $\mathrm{N}_{2}$ and $\mathrm{CO}_{2}$, and feedstock on microbial community in metal(loid)s contaminated soils. Environ. Int. 2019, 126, 791-801. [CrossRef] [PubMed]

9. Sultan, U.; Zhang, Y.; Farooq, M.; Imran, M.; Khan, A.A.; Zhuge, W.; Khan, T.A.; Yousaf, M.H.; Ali, Q. Qualitative assessment and global mapping of supercritical $\mathrm{CO}_{2}$ power cycle technology. Sustain. Energy Technol. Assess. 2021, 43, 100978. [CrossRef]

10. Godin, J.; Liu, W.; Ren, S.; Xu, C.C. Advances in recovery and utilization of carbon dioxide: A brief review. J. Environ. Chem. Eng. 2021, 9, 105644. [CrossRef]

11. Siddiqui, M.A.Q.; Ueda, K.; Komatsu, H.; Shimamoto, T.; Roshan, H. Caveats of using fractal analysis for clay rich pore systems. J. Pet. Sci. Eng. 2020, 195, 107622. [CrossRef] 
12. Li, C.; Hao, M.; Geng, Z.; He, Y.; Wei, S. Drop-weight impact fragmentation of gas-containing coal particles. Particuology 2021, 55, 35-42. [CrossRef]

13. Jiang, G.; Cai, L.; Wang, S.; Laaksonen, A.; Feng, X.; Mu, L.; Lu, X.; Zhu, J. Critical role of carbonized cellulose in the evolution of highly porous biocarbon: Seeing the structural and compositional changes of spent mushroom substrate by deconvoluted thermogravimetric analysis. Ind. Eng. Chem. Res. 2020, 59, 22541-22548. [CrossRef]

14. Ossler, F.; Santodonato, L.J.; Bilheux, H.Z. In-situ neutron imaging of hydrogenous fuels in combustion generated porous carbons under dynamic and steady state pressure conditions. Carbon 2017, 116, 766-776. [CrossRef]

15. Geng, Z.; Xiao, Q.; Lv, H.; Li, B.; Wu, H.; Lu, Y.; Zhang, C. One-step synthesis of microporous carbon monoliths derived from biomass with high nitrogen doping content for highly selective $\mathrm{CO}_{2}$ capture. Sci. Rep. 2016, 6, 30049. [CrossRef]

16. Cai, J.; Lin, D.; Singh, H.; Wei, W.; Zhou, S. Shale gas transport model in 3D fractal porous media with variable pore sizes. Mar. Pet. Geol. 2018, 98, 437-447. [CrossRef]

17. Zhang, R.-H.; Wu, J.-F.; Zhao, Y.-L.; He, X.; Wang, R.-H. Numerical simulation of the feasibility of supercritical $\mathrm{CO}_{2}$ storage and enhanced shale gas recovery considering complex fracture networks. J. Pet. Sci. Eng. 2021, 204, 108671. [CrossRef]

18. He, H.; Liu, P.; Xu, L.; Hao, S.; Qiu, X.; Shan, C.; Zhou, Y. Pore structure representations based on nitrogen adsorption experiments and an FHH fractal model: Case study of the block Z shales in the Ordos Basin, China. J. Pet. Sci. Eng. 2021, 203, 108661. [CrossRef]

19. Zhang, L.; Cao, S.; Li, Z.; Zhang, H.; Duan, F. Pore structure of sludge char and simultaneously NO removal characteristics in the treated flue gas from Ca-L. Fuel 2021, 289, 119937. [CrossRef]

20. Xiang, G.; Ye, W.; Yu, F.; Wang, Y.; Fang, Y. Surface fractal dimension of bentonite affected by long-term corrosion in alkaline solution. Appl. Clay Sci. 2019, 175, 94-101. [CrossRef]

21. Feng, Y.; Li, Y.; Wang, J.; Wu, M.; Fan, H.; Mi, J. Insights to the microwave effect in the preparation of sorbent for $\mathrm{H}_{2} \mathrm{~S}$ removal: Desulfurization kinetics and characterization. Fuel 2017, 203, 233-243. [CrossRef]

22. Li, Z.; Liu, D.; Cai, Y.; Wang, Y.; Teng, J. Adsorption pore structure and its fractal characteristics of coals by $\mathrm{N}_{2}$ adsorption/desorption and FESEM image analyses. Fuel 2019, 257, 116031. [CrossRef]

23. Wen, H.; Wei, G.; Ma, L.; Li, Z.; Lei, C.; Hao, J. Damage characteristics of coal microstructure with liquid $\mathrm{CO}_{2}$ freezing-thawing. Fuel 2019, 249, 169-177. [CrossRef]

24. Ren, Q.; Zhang, Y.; Arauzo, I.; Shan, L.; Xu, J.; Wang, Y.; Su, S.; Hu, S.; Xiang, J. Roles of moisture and cyclic loading in microstructures and their effects on mechanical properties for typical Chinese bituminous coals. Fuel 2021, 293, 120408. [CrossRef]

25. Lin, K.; Huang, X.; Zhao, Y.-P. Combining image recognition and simulation to reproduce the adsorption/desorption behaviors of shale gas. Energy Fuels 2020, 34, 258-269. [CrossRef]

26. Panigrahi, S.; Sharma, H.B.; Dubey, B.K. Overcoming yard waste recalcitrance through four different liquid hot water pretreatment techniques-Structural evolution, biogas production and energy balance. Biomass Bioenergy 2019, 127, 105268. [CrossRef]

27. Iftikhar, M.; Asghar, A.; Ramzan, N.; Sajjadi, B.; Chen, W.-Y. Biomass densification: Effect of cow dung on the physicochemical properties of wheat straw and rice husk based biomass pellets. Biomass Bioenergy 2019, 122, 1-16. [CrossRef]

28. Sharma, H.B.; Dubey, B.K. Co-hydrothermal carbonization of food waste with yard waste for solid biofuel production: Hydrochar characterization and its pelletization. Waste Manag. 2020, 118, 521-533. [CrossRef] [PubMed]

29. Whittaker, C.; Shield, I. Factors affecting wood, energy grass and straw pellet durability-A review. Renew. Sustain. Energy Rev. 2017, 71, 1-11. [CrossRef]

30. Goel, C.; Kaur, H.; Bhunia, H.; Bajpai, P.K. Carbon dioxide adsorption on nitrogen enriched carbon adsorbents: Experimental, kinetics, isothermal and thermodynamic studies. J. $\mathrm{CO}_{2}$ Util. 2016, 16, 50-63. [CrossRef]

31. Gargiulo, V.; Gomis-Berenguer, A.; Giudicianni, P.; Ania, C.O.; Ragucci, R.; Alfè, M. Assessing the potential of biochars prepared by steam-assisted slow pyrolysis for $\mathrm{CO}_{2}$ adsorption and separation. Energy Fuels 2018, 32, 10218-10227. [CrossRef]

32. Abdulmumini, M.M.; Zigan, S.; Bradley, M.S.; Lestander, T.A. Fuel pellet breakage in pneumatic transport and durability tests. Renew. Energy 2020, 157, 911-919. [CrossRef]

33. Sigvardsen, N.M.; Ottosen, L.M. Characterization of coal bio ash from wood pellets and low-alkali coal fly ash and use as partial cement replacement in mortar. Cem. Concr. Compos. 2019, 95, 25-32. [CrossRef]

34. Panigrahy, C.; Seal, A.; Mahato, N.K.; Bhattacharjee, D. Differential box counting methods for estimating fractal dimension of gray-scale images: A survey. Chaos Solitons Fractals 2019, 126, 178-202. [CrossRef]

35. Tjørve, K.M.C.; Tjorve, E. The use of Gompertz models in growth analyses, and new Gompertz-model approach: An addition to the Unified-Richards family. PLoS ONE 2017, 12, e0178691. [CrossRef] [PubMed]

36. Migenda, N.; Möller, R.; Schenck, W. Adaptive dimensionality reduction for neural network-based online principal component analysis. PLOS ONE 2021, 16, e0248896. [CrossRef]

37. R Core Team. R: A Language and Environment for Statistical Computing. Available online: http://www.R-project.org/ (accessed on 25 June 2021).

38. Sridhar, M.; Gunugunuri, K.; Hu, N.; Motahari, A.; Zuo, X.; Schaefer, D.W.; Thiel, S.W.; Smirniotis, P.G. Impact of interfacial roughness on the sorption properties of nanocast polymers. Macromolecules 2016, 49, 2663-2670. [CrossRef] 